\title{
Les Roms, révélateurs des anomalies d'un « Espace de liberté, de sécurité et de justice au service des citoyens »
}

Anaïs Faure Atger et Alejandro Eggenschwiler

\section{(2) OpenEdition \\ Journals}

Édition électronique

URL : http://journals.openedition.org/conflits/18148

DOI : $10.4000 /$ conflits. 18148

ISSN : $1777-5345$

Éditeur :

CCLS - Centre d'études sur les conflits lilberté et sécurité, L'Harmattan

Édition imprimée

Date de publication : 5 septembre 2011

Pagination : 195-201

ISBN : 978-2-296-56086-4

ISSN : 1157-996X

Référence électronique

Anaïs Faure Atger et Alejandro Eggenschwiler, « Les Roms, révélateurs des anomalies d'un «Espace de liberté, de sécurité et de justice au service des citoyens » », Cultures \& Conflits [En ligne], 81-82 | Printemps/Été 2011, mis en ligne le 05 septembre 2012, consulté le 30 mars 2021. URL : http:// journals.openedition.org/conflits/18148; DOI : https://doi.org/10.4000/conflits.18148 


\section{Les Roms, révélateurs des anomalies d'un « Espace de liberté, de sécurité et de justice au service des citoyens »}

\section{Anaïs FAURE ATGER et Alejandro EGGENSCHWILER}

Anaïs Faure Atger est Chargée de recherche dans la section Justice et Affaires intérieures an Centre for European Policy Studies (CEPS). Alejandro Eggenschwiler est Assistant de recherche dans la même section.

$\mathrm{E}$ n 1999, le Traité d'Amsterdam a établi les fondations de l'Espace européen de liberté, de sécurité et de justice (ELSJ). Celui-ci prévoit l'harmonisation des lois dans les domaines de l'immigration, de l'asile, du contrôle des frontières et des visas, à l'appui du principe de la libre circulation des personnes. Le programme de Stockholm, adopté en décembre 2009, définit les orientations stratégiques des politiques européennes correspondantes pour les cinq prochaines années. Il entend placer les citoyens au cœur des politiques dans ce domaine. Ces engagements européens en faveur de la protection des droits de l'individu ont d'ailleurs été réaffirmés par l'intégration de la Charte des droits fondamentaux dans le droit de l'Union suite à l'adoption du Traité de Lisbonne. Toutefois, ces velléités d'intégration européenne autour de principes communs, tels que l'interdiction de toute forme de discrimination et de respect des droits fondamentaux, se heurtent à la résistance des États membres face au transfert de compétences qui, jusqu'ici, étaient jalousement décidées à la seule discrétion du pouvoir exécutif. Un des aspects les plus manifestes d'une telle résistance consiste à réduire la portée des lois et des protections conférées par la législation européenne au moment de sa transposition au niveau national. En général, ce sont les plus vulnérables, les ressortissants de pays tiers et les minorités qui en sont les premières victimes.

Avec plus de six millions de membres ${ }^{1}$, les Roms représentent la minorité ethnique la plus importante d'Europe. En dépit de la place qu'ils occupent, la

1. Le Conseil de l'Europe estimait en septembre 2009 que le nombre de Roms résidant dans l'Union européenne se situait entre 4359100 et 7456 500. Voir les statistiques préparées par le département "Roms et Gens du Voyage » du Conseil de l'Europe. http://www.coe.int/t/dg3/romatravellers/default_FR.asp. Page consultée le 26 mai 2011. 
situation des communautés Roms en Europe pâtit depuis longtemps des phénomènes suivants : violation des droits, stigmatisation, préjugés et exclusion sociale. Devenus pour la plupart citoyens européens, ils sont néanmoins en butte à toutes sortes de discriminations raciales et sociales. Le présent article fait ressortir les contradictions que présentent les acquis de l'ELSJ et la manière dont la question rom est traitée par certains États membres. Différents aspects sont passés en revue : tout d'abord, les dimensions internes de l'ELSJ, et plus particulièrement le droit à la libre circulation, caractérisé par l'interdiction de tout forme de discrimination et l'abolition des contrôles physiques aux frontières ; puis, les éléments affectant les politiques communes en matière d'asile et de visas sont analysés au regard de leurs conséquences sur la politique extérieure de l'Union. L'article conclut en faisant valoir que la situation actuelle des Roms en Europe montre les anomalies d'un espace de liberté, de sécurité et de justice entre des États souverains qui sont réticents à faire profiter de la même manière la population et les capitaux du marché commun européen, et nuit ainsi à la légitimité tant interne qu'externe de l'Union.

\section{Une liberté de circulation limitée}

C'est en Roumanie, en Bulgarie, en Espagne et en Hongrie que la présence des Roms est la plus importante. En tant que citoyens de pays membres de l’UE, ils devraient bénéficier des mêmes droits et responsabilités que tout autre citoyen européen, et notamment de la liberté de circulation et de résidence. La protection des citoyens d'un État membre contre l'expulsion ou l'éloignement du territoire d'un autre État membre est une caractéristique essentielle de la citoyenneté européenne. Toute décision de renvoi d'un citoyen européen vers son pays d’origine doit être évaluée au cas par cas, au regard des principes de la liberté de circulation, de l'égalité et de la non-discrimination énoncés dans les traités, la Charte des droits fondamentaux de l'Union et la directive européenne relative au droit des citoyens de l'Union et des membres de leurs familles de circuler et de séjourner librement sur le territoire des États membres.

Au cours de l'été 2010, le gouvernement français a publié des circulaires administratives appelant au démantèlement de camps illégaux et à l'expulsion immédiate de leurs occupants. Selon ces instructions qui émanaient des plus hautes sphères de l'État, elles devaient être appliquées en priorité à l'encontre des populations Roms. Le débat houleux qui suivit leur publication a opportunément été orienté par le gouvernement français sur la légitimité du droit de regard de la Commission et de la Commissaire européenne en charge de la justice, des droits fondamentaux et de la citoyenneté Vivianne Reding, dans les affaires internes de la France ${ }^{2}$. Néanmoins, vu la nature des consignes administratives françaises, la question aurait dû être de savoir dans quelle mesure ces instructions étaient conformes au droit européen et à la Charte européenne, notamment au principe de non-discrimination. D’après les traités 
européens, c'est à la Commission qu'il incombe de veiller à l'application correcte du droit de l'Union. Le recours en manquement prévu à l'article $258 \mathrm{du}$ Traité sur le fonctionnement de l'Union est l'outil dont elle dispose pour tenter de mettre fin aux infractions éventuelles constatées dans un État membre. Il donne au Collège des Commissaires - tout en laissant une telle compétence à sa discrétion - le pouvoir d'examiner et d'engager une procédure d'infraction à l'encontre d'un État membre « récalcitrant » soupçonné de manquer à ses obligations européennes.

En dépit de ce rôle reconnu par les traités de « contrôleur » de la conformité des pratiques nationales avec le droit communautaire, la Commission s'est finalement résignée le 19 octobre 2010 à ne pas faire usage de ce pouvoir. Suite à la promesse française de transposer correctement la directive relative aux droits des citoyens de l'Union, la procédure à l'encontre de la France a donc été abandonnée. Ce renoncement, certainement du à de fortes pressions politiques au sein même de la Commission, a eu des conséquences désastreuses sur la légitimité de l'architecture européenne. Une telle attitude n'a pas uniquement mis en péril le droit à la libre circulation des citoyens Roms. Elle a aussi encouragé le gouvernement français à opposer aux légitimes incriminations de la Commission et du Parlement européen, la «grandeur » d'un des États membres.

\section{Interruption du processus d'abolition des frontières}

La condition sine qua non de la liberté de circulation des biens et des personnes est l'abolition des contrôles aux frontières intérieures. L'accord Schengen conclu le 14 juin 1985 en a défini les principes de base. Il a été mis en application par vagues successives dans les États membres, et comprend aujourd'hui vingt-cinq États membres. La Roumanie (1,8 million de Roms) et la Bulgarie (750 000 Roms) sont les prochains en lice. Avant d'obtenir le feu vert pour l'espace sans frontières, les nouveaux États membres doivent d'abord se conformer à l'intégralité des acquis Schengen ${ }^{3}$. Une coopération policière renforcée et le contrôle des frontières extérieures de l'Union sont considérés comme des étapes préalables à la libre circulation. Des évaluations techniques et des visites d'inspection coordonnées par la Commission européenne sont prévues pour vérifier la bonne mise en œuvre de cette collaboration. La décision finale revient ensuite au Conseil, après examen de la situation des pays candidats. Jusqu'en 2007, le processus conduisant à l'abolition des frontières intérieures s'attachait essentiellement à vérifier l'état de protec-

2. Carrera S., Faure Atger A., L'Affaire des Roms: A Challenge to the EU's Area of Freedom, Security and Justice, CEPS Liberty and Security in Europe, septembre 2010. Texte disponible à l'adresse suivante: http://www.ceps.eu/book/l\%E2\% 80\%99affaire-des-roms-challengeeu\%E2\% 80\% 99s-area-freedom-security-and-justice. Page consultée le 26 mai 2011.

3. Faure Atger A., The Abolition of Internal Border Checks in an Enlarged Schengen Area: Freedom of movement or a scattered web of security checks?, Challenge Paper, mars 2008. 
tion de la frontière extérieure et la bonne intégration des mécanismes de coopération policière. Ces évaluations Schengen se sont progressivement étendues et, pour la Bulgarie et la Roumanie, des critères complémentaires semblent maintenant être pris en compte.

Jusqu'à présent, le processus d'abolition des contrôles aux frontières exercés sur les personnes survenait peu de temps après que les pays candidats soient connectés au Système d'information Schengen, la base de données informatique regroupant des informations judiciaires et criminelles sur les personnes et les biens, que la Roumanie et la Bulgarie ont intégrée en juin 2010. En dépit de l'agenda fixant à 2011 l'abolition des contrôles sur les personnes entre ces pays et le reste de l'Union, deux États membres ont, en décembre 2010, informé la Commission par courrier qu'ils ne donneraient pas leur accord ${ }^{4}$. Tout en justifiant leur décision par des arguments divers tels que la corruption généralisée et l'existence d'un conflit frontalier latent entre la Bulgarie et la Turquie, d'autres considérations de caractère plus politique ont certainement joué un rôle, donnant à penser que l'on assistait à un règlement de compte plutôt qu'à une décision basée sur des critères strictement définis. Au cours de l'année précédente, les deux États membres qui ont exprimé ce veto, à savoir la France et l'Allemagne, s'étaient en effet livrés au renvoi de milliers de Roms vers les pays en question. La perspective de la libre circulation de ces citoyens au sein de l'Union apparaît donc avoir été considérée comme un critère supplémentaire. De fait, les parlementaires européens ont depuis lors exprimé leur inquiétude face au manque de transparence de cette décision et aux considérations politiques qui ont pu la motiver. Le président roumain s'est dit déçu de ce manque de solidarité. La complaisance dont il avait fait preuve à l'égard du gouvernement français alors que celui-ci expulsait collectivement ses citoyens avait alors été expliquée par son souci d'obtenir l'unanimité au Conseil sur ces questions, quelques mois plus tard. Les Roms ont ainsi été à nouveau propulsés sur le devant de la scène européenne, perturbant le mécanisme d'intégration pour la libre circulation des personnes et démontrant l'importance des relations bilatérales au sein de l'ESLJ.

\section{Le système européen d'asile mis à mal}

Depuis 1999, l'Union européenne s'est engagée dans un processus d'harmonisation des lois dans le domaine de l'asile et de la protection des réfugiés. Une telle entreprise inclut des mesures relatives aux conditions de réception des demandeurs et aux procédures d'octroi et de retrait du statut de réfugié, ainsi que le mécanisme dit de Dublin, qui détermine l'État membre responsable de l'examen de la demande et des procédures applicables dans ce domaine. Le principe qui est au cœur du système européen d'asile consiste à présumer que les droits fondamentaux sont respectés à tout moment et en tous lieux au

4. AFP, «Schengen : Bucarest appelle à ne pas “changer les règles du jeu” ", 3 janvier 2011 
sein de l'Union. De ce fait, il ne peut y avoir de demandeurs d'asile originaires d'un des États membres. Aux termes du Protocole sur le droit d'asile pour les ressortissants des États membres de l'Union européenne (dit Protocole Aznar), et vu le niveau de protection des droits fondamentaux et des libertés fondamentales dans les États membres de l'Union, ceux-ci sont considérés comme constituant des pays d'origine sûrs les uns vis-à-vis des autres pour toutes les questions pratiques et juridiques liées aux affaires d'asile. En conséquence, toute demande d'asile présentée par un ressortissant d'un État membre ne peut être prise en considération ni déclarée admissible pour instruction par un autre État membre.

En 2008, la Commission de l'immigration et du statut de réfugié du Canada a accordé le statut de réfugié à quatre-vingt-quatre citoyens européens de nationalité tchèque ${ }^{5}$. Sur les cent-quatre-vingt-quatorze demandes d'asile reçues, 43 \% ont été considérées comme justifiées. De même, en 2009, quatrevingt-dix demandes de protection sur cent-soixante-six ont été agréées, soit un taux de reconnaissance de $54 \%$. Les Roms, car ils l'étaient tous, sont donc contraints de chercher protection hors d'Europe, face aux discriminations et aux persécutions dont ils sont victimes dans leur pays d'origine. Force est de constater que le système européen d'asile méconnaît la situation des citoyens européens d'origine rom dans de nombreux États membres et qu'il ne permet pas à l'Union d'assurer la protection de ses propres citoyens. Espérant contenir cet afflux et forcer l'Union à réagir, le Canada décide en juillet 2009 de réintroduire l'obligation de visa pour les ressortissants tchèques. Suite à l'adhésion de la République tchèque à l'Union européenne, les visas n'étaient en effet plus requis. Alors qu'elle était confrontée à son incapacité à protéger ses ressortissants contre ce qu'un État tiers assimile à une persécution, l'Union s'est contentée de négocier le retour de la réciprocité. Elle a servi de plateforme pour tenter d'obtenir la levée de ces restrictions au voyage, au lieu de remédier aux raisons qui poussaient ces Roms à l'exil canadien. Cette affaire jetait pourtant de sérieux doutes quant au respect par un État membre de ses obligations internationales en matière de droits de l'homme et mettait à mal les principes fondateurs du système européen d'asile.

\section{Incohérences de la politique commune des visas}

La compétence de l'Union en ce qui concerne la politique des visas se limite aux visas de courte durée, d'une durée maximale de trois mois. Cette politique commune s'inscrit dans une stratégie globale de protection des frontières extérieures à l'espace Schengen. Elle concerne tous les États membres

5. Eggenschwiler A., The Canada-Czech Republic Visa Affair: A test for visa reciprocity and fundamental rights in the European Union, CEPS Liberty and Security in Europe, novembre 2010. Texte disponible à l'adresse suivante: http://ceps.be/book/canada-czech-republic-visaaffair-test-visa-reciprocity-and-fundamental-rights-european-union. Page consultée le 26 mai 2011. 
ayant aboli les contrôles aux frontières intérieures et appliquant les acquis Schengen. Suite au transfert de compétence à l'Union en 1999, l'adoption de nombreux instruments a contribué à façonner des principes et règles communs dans ce domaine. Un code des visas a été adopté énonçant des procédures et des critères communs à tous les États membres pour l'octroi du sésame donnant accès au territoire européen. Différents règlements du Conseil définissent aussi une liste de pays tiers dont les ressortissants sont soumis à cet instrument de " mise à l'écart " (la liste noire) et celle des autres pays (la liste blanche). Un mécanisme européen permet enfin aux États membres de réagir de concert lorsqu'un État de la liste blanche restaure une obligation de visa à un citoyen européen. Ce mécanisme a pour objet de faciliter le dialogue entre les parties afin d'identifier la cause du différend, de le résoudre de concert ou de réintroduire l'obligation de visa Schengen pour les citoyens de l'État tiers concerné. La Commission européenne et le Conseil sont les principaux acteurs de la mise en ouvre du principe de réciprocité des visas, aspect clef de la politique européenne commune en la matière.

L'Union européenne, qui se perçoit comme un espace intégré, attend des pays tiers que tous les États membres soient traités de manière identique. Depuis l'élargissement de 2004, l'Union s'attache à obtenir des obligations de visa similaires pour tous les citoyens européens. Un des aspects essentiels de la politique extérieure de l'UE envers le Canada consistait à étendre l'exemption de visa aux ressortissants des nouveaux États membres, ce jusqu'à ce que des Roms de ces pays soient considérés comme victimes de persécutions. La réintroduction des visas représente donc un recul de la coopération entre l'Union européenne et le Canada. C'est aussi la première fois qu'un État de la liste blanche décide de rétablir de manière unilatérale l'obligation de visa à l'égard d'un État membre de l'Union. Or le principe de réciprocité, qui devait censément permettre de faire face à de telles situations, s'est révélé inadapté. La majorité des États membres a en effet préféré ne pas se risquer à imposer à nouveau l'obligation de visa aux ressortissants canadiens de crainte de s'exposer au même traitement. La discrimination dont les Roms font l'objet dans certains États membres menace donc la politique commune des visas et révèle les asymétries de la politique extérieure de l’UE.

\section{Conclusion}

La situation des Roms dans l'Union européenne s'inscrit en opposition avec les principes sur lesquels l'Union prétend se fonder. Victimes de discrimination, d'inégalités, de stigmatisation et de persécution, les Roms illustrent les limites d'un Espace européen de liberté, de sécurité et de justice au service de ses citoyens. Restrictions à la liberté de circulation, remise en question du système européen d'asile, brèches dans la politique commune de visas sont autant d'éléments qui révèlent les anomalies de cette construction politique commune. De même, la résistance des États membres à l'européanisation dans 
certains domaines, la remise en cause de l'architecture européenne, le choix « à la carte » des bénéficiaires des droits et protections prévus dans les traités européens sont autant d'indicateurs qui témoignent de l'incapacité institutionnelle de l'Union à faire respecter les droits de l'homme et à protéger les citoyens européens contre l'arbitraire des forces au pouvoir au niveau national. Face à cette absence évidente de volonté politique de protéger les citoyens européens les plus vulnérables, il serait pourtant plus que jamais nécessaire à l'Europe de se positionner en tant que défenseur des droits de l'homme si elle veut conserver sa légitimité auprès des Européens et sa crédibilité sur la scène internationale. 